INVESTIGACIÓN/RESEARCH

\title{
ASPECTOS SOCIALES Y SOCIOLÓGICOS EN EL ESTRÉS DE LOS DOCENTES
}

Nuria Arís Redó1: Universidad Internacional de Cataluña. España.

nuria@uic.es

\section{RESUMEN}

El estrés docente es un proceso que se desarrolla como respuesta a las presiones en el ámbito laboral. El profesor en su acción cotidiana se ve inmerso en la dinámica de asimilar las profundas transformaciones que se han producido en la enseñanza, en el aula y en el contexto social que lo rodea. Al hablar del estrés social hacemos referencia al estrés colectivo, al de los grupos, al de la sociedad en general que se proyecta en la cotidianidad del aula y de los maestros en general. En este artículo, presentamos los factores más destacados en el origen social y sociológico del estrés docente. Así pues analizamos y reflexionamos sobre aspectos como la consideración de sociedad y el binomio red-sistema educativo, el fenómeno social de la educación en sí mismo y sus funciones, la crisis social y de valores, etc.

PALABRAS CLAVE: Estrés - Estrés docente - Estrés social.

\footnotetext{
${ }^{1}$ Autor correspondiente:
}

Nuria Arís Redó: Profesora Agregada Facultad de Educación de la Universidad Internacional de Cataluña. España.

Correo: nuria@uic.es 


\title{
THE SOCIAL AND SOCIOLOGICAL ASPECTS OF THE TEACHING STRESS
}

\begin{abstract}
Teaching stress is a process developed as a response to the pressure of the labour environment. On their daily routine, teachers get involved in the dynamics of assimilating the deep transformations education has experienced, as well as classes and social context have, too. When talking about social streets, we refer to collective stress, group stress, social stress in general. In this article, our aim is to analyse and think about the stressors of the social stress itself. We are showing those factors most related to the social and sociological aspects of the teaching stress. We are analysing and reflecting about aspects as the consideration of society and the pairing neteducational system, the social educational teaching phenomena itself and its purposes, the social crisis and values, etc.
\end{abstract}

KEY WORDS. Stress - Teaching stress - Social stress.

\section{INTRODUCCIÓN}

Este artículo pretende reflexionar sobre determinados aspectos vinculados al estrés social y de los colectivos que inciden en la configuración del estrés en los docentes. La realidad personal y la realidad social se retroalimentan desde la interacción de las personas, entre ellas y con su contexto. Debemos entender la escuela como un sistema abierto que se relaciona íntimamente con su entorno y donde la relación entre la comprensión y la acción forman una espiral dialéctica donde ambas se potencian mutuamente. Las posibles causas del elevado riesgo de sufrir tensión y estrés en el entorno docente son muy diversas y ciertamente complejas.

Por ello, pretendemos analizar entre otros aspectos: la imagen social del maestro y los cambios sociales que han tenido su reflejo en el mundo educativo, la crisis de valores o la falta de referentes, globalización, etc.

\section{OBJETIVOS}

Los objetivos específicos son:

1.-Analizar y reflexionar sobre aspectos como el binomio red-sistema educativo, el fenómeno social de la educación en sí mismo y sus funciones, la crisis social y de valores.

2.- Reflexionar sobre dichas dimensiones considerando un enfoque global.

3.- Comprender las dimensiones objeto de estudio y a partir de ellas establecer las relaciones más significativas para su interpretación.

\section{METODOLOGÍA}


A partir de una metodología cualitativa-interpretativa se pretende estudiar en profundidad una situación concreta para facilitar un análisis descriptivo e interpretativo. El paradigma cualitativo orientado a una investigación interpretativa pretende describir descubrir pautas, patrones y regularidades. Partimos de una metodología cualitativa y orientada a la comprensión, para describir e interpretar la relación entre dichos aspectos, y su repercusión en el entorno más inmediato. La técnica para la recogida de información consiste en una rigurosa revisión de la literatura científica.

\section{RESULTADOS}

\subsection{El estres social en el ámbito educativo.}

Nuestro punto de partida se basa en considerar que las realidades individuales y sociales son interdependientes y su explicación e interpretación también resulta inseparable. Nos referimos a relaciones de mutua interdependencia. Por eso no se puede explicar el comportamiento de cada una de esas realidades sin considerar además el de las personas, los elementos materiales, de control y el funcionamiento del conjunto, así como sus conexiones con otros sistemas externos con los que se interactúa. La psicología de los grupos tiene rasgos distintivos propios que inciden en la percepción del estrés, Shaw (1983), Canto (1998), Gil y otros (1999). Por el contrario, el estrés social en el ámbito educativo es una temática que tiene muy poca bibliografía en comparación a los materiales del estrés personal, Sol (2009), Gracia-Herrero-Musitu (2002).

Presentamos los factores más destacados de una larga lista de factores descritos en relación al estrés docente, Riart, J. Coord. (2013). Queremos destacar aquellos más vinculados a la realidad más social y sociología del estrés docente.

\subsection{Los sistemas educativos, el binomio Sociedad-Red.}

Morin (1999a, 1999b) explica que, para que la educación pueda reformar los espíritus y la sociedad en general, primero hay que reformar la educación. Define su pensamiento como co-constructivismo, queriendo decir que se precisa la colaboración de nuestro intelecto y de la realidad exterior para construir conocimiento. El año 1999 publicó, en colaboración con la UNESCO, Los siete saberes necesarios para la educación del futuro, que simplificando mucho, son éstos:

1.- Enfrentar las cegueras del conocimiento: el error y la ilusión.

2.- Alimentar los principios de un conocimiento pertinente.

3.- Enseñar la condición humana.

4.- Enseñar la identidad de la tierra.

5.- Hacer frente a las incertidumbres.

6.- Enseñar la comprensión.

7.- Cultivar la ética del género humano. 
Estos siete saberes no se contradicen en absoluto con las tesis de su última obra; a pesar de haber pasado once años desde su publicación, todavía son vigentes Morin (2008).

Por otro lado, para Manel Castells $(2000,2002,2009)$ con Internet se rompe la idea de una educación basada en la "transmisión de paquetes de conocimientos acumulados" para convertirse en un aprendizaje de cómo ser capaz de acceder en la red, como obtener el máximo provecho y sobre todo, como utilizar el gran volumen de datos accesibles. Hay que aprender a seleccionar la información y a proporcionar instrumentos para procesarla. Pero, ¿cómo hacerlo? La sociedad-red plantea grandes retos en el mundo educativo, donde forzosamente, el impacto de las nuevas tecnologías hace cambiar las maneras de enseñar y de aprender, y lo cambiarán todavía más en el futuro. Estamos ante una verdadera revolución social y educativa. Este nuevo paradigma educativo provoca que el docente no sepa exactamente como "enseñar" a sus alumnos y evidentemente acentúa el estrés, el personal, el de su colectivo y el estrés social general del que ahora hablamos.

Los sistemas educativos son piezas fundamentales en el mantenimiento de una organización social y, por lo tanto, transmisores de ideología. No existe la educación neutra. La educación moral o la educación en valores es muy compleja, Morin (2001), Riart (2011), por eso requiere que participen diferentes agentes de socialización, con varias estrategias educativas y adaptándose al contexto concreto. La tarea consciente de los profesores puede contribuir a la mecánica de reproducción social o a la resistencia a ella. Pero la escuela no es más que uno de los agentes socializadores. La escuela puede hacer, y debe hacer el papel de introducir la reflexión crítica sobre la orden convencional moral y social. Si la educación quiere promover cambios sociales lo que tiene que hacer es educar personas: participativas, independientes, críticas, flexibles y creativas.

\subsection{Crisis social. Valores "líquidos".}

Hay una crisis de valores, unas generaciones nuevas con referentes mentales y laborales diferentes y actitudes sociales diferentes. La corrupción está a la orden del día, también de manera reiterada. Las intolerancias, derivadas de la permisividad son criterios actitudinales. Esto se constituye en estrés de los docentes y al estrés social, no solamente por las personas que han vivido y viven estos cambios, sino también por las nuevas generaciones que en este punto, especialmente, son erráticas en normas, actitud e incluso valores sociales y laborales. Hablamos de manera global, a pesar de que hay subgrupos de personas con criterios claros. Pero a nivel colectivo es un elemento más que contribuye al aumento de la presión y estrés social en el ámbito escolar. Estamos en una sociedad "dinámica", es decir, cambiante e incierta.

Para hacer una breve aproximación a las características de la sociedad actual, hemos escogido, entre muchas posibilidades, tres autores del ámbito de la sociología, cercanos a nuestro entorno: Zygmunt Bauman (2007b), Manuel Castells (2007) y Edgar Moran, (2001, 2008). Tomamos como principal referencia las obras más recientes de estos autores.Bauman habla de una sociedad "líquida", en la cual casi todo parece 
inestable, inseguro y amenazando. Castells se refiere a la sociedad "red" para designar un nuevo tipo de sociabilidad, centrada en las TIC, el alcance y las consecuencias de la cual todavía no podemos prever. Según Bauman (2007), la educación de hoy tiene diferentes retos, entre los que destaca:

a) Las migraciones. Para *Bauman, la diáspora es un modelo social de adaptación que nos trae a vivir y convivir con la diferencia. Entonces hablamos de educación intercultural, basada en el diálogo, el entendimiento y la convivencia y donde la escuela puede acontecer un crisol de culturas.

b) La cultura en la sociedad líquida. El modelo actual se fundamenta en las elecciones individuales que se mueven por la seducción, los deseos y las relaciones públicas. No hay un modelo cultural establo, sino muchas opciones culturales como consecuencia de la diáspora y de la globalización. Y esto choca de pleno con el tema de la integración cultural y de la cohesión social.

c) La comunicación intergeneracional. Los niños viven en un mundo que en pocos años ha acontecido radicalmente diferente del de sus padres y todavía más del que vivieron sus abuelos. Hay que recordar el que decimos de una generación cada cinco años. Hay un mundo virtual, on-line y otro mundo off-line (real) con reglas diferentes. Los cambios tecnológicos adoptan una velocidad de vértigo. El concepto de "largo plazo" se ha devaluado. El "Kleenex" fue el primer ejemplo de la cultura basada al "utilizar y tirar", que después ha pasado al teléfono móvil e incluso a las relaciones de pareja, para poner algunos ejemplos.

\subsection{El fenómeno social de la educación: reorientación de sus funciones.}

Para que una sociedad sea viable, y por lo tanto no genere un estrés social preocupante, debe haber un grado de socialización suficiente que haga posible la vida colectiva. Socializar -se quiere decir aprender y aprender la cultura de la sociedad a la cual, por nacimiento, pertenecemos. La mayor parte de la conducta humana es aprendida y la socialización es el proceso mediante el cual los individuos aprenden e interiorizan las formas normales de vida de una comunidad. La sociedad se reproduce a lo largo del tiempo y los individuos se introducen en la historia. La socialización convierte un individuo generado biológicamente en un ser social y cultural, se aprenden los roles, se asumen normas, se desarrollan actitudes. La sociedad no es un simple conglomerado de individuos, sino que es un sistema estructurado como resultado de las diferentes relaciones establecidas entre los diferentes grupos sociales. La sociedad está estructurada por relaciones asimétricas, los grupos dominantes son los que tienen la capacidad efectiva para imponer su visión de la realidad (que nace de la defensa de sus intereses como grupo) a la del resto de los grupos sociales. La socialización comporta:

-Proceso de adquisición de la cultura (primera infancia).

-Proceso de integración de la cultura dentro de la personalidad (hace que no nos damos cuenta del control social: función liberadora / objeto de coacción social).

-Proceso de adaptación del individuo al entorno social (sentimiento de pertenencia: integración individuo / perdurabilitat colectivo).

Dentro de una sociedad hay rasgos universales comunes y rasgos particulares de cada grupo diferenciado. Algunos se denominan básicos y se los concede gran importancia. 
Esos rasgos básicos acostumbran a referirse a la familia, la religión, la lengua y a las costumbres socialmente establecidas. La educación es un tipo particular de socialización. Estamos hablando de un sistema de supervivencia de la sociedad. La educación es un fenómeno social por los siguientes motivos:

- Por el medio en que se efectúa, que es un medio social.

- Por los contenidos que constituyen su objeto propio (cultura social).

- Por los fines de esta educación, muchos de los cuales son sociales.

- Por las funciones sociales que le son inherentes.

- Por los factores que la motivan y realizan.

- Por los acondicionamientos a que se ve sujeta, sociales en su mayoría.

Hay que considerar con qué mecanismos colabora el sistema educativo en la continuidad vital del sistema social instaurado. Althusser (1968), de aparato ideológico del Estado (familia, escuela, medios de comunicación...). También Baudelot y Establet (1976) afirman que la escuela de las sociedades capitalistas es el instrumento principal para mantener y reproducir las relaciones sociales de producción establecidas al si de la estructura económica entre explotadores y explotados. Bowles y Gintis (1981) hablan de como el sistema educativo prepara futuros trabajadores mediante la correspondencia entre las relaciones sociales de producción y las relaciones sociales escolares. A su vez, Bourdieu y Passeron $(1973,1977)$ afirman que la institución escolar valora un tipo de aptitudes y actitudes que corresponden a la clase alta, hecho que causa que la selección escolar acontezca "la elección de los elegidos".

En cualquier caso, la educación cumple una función al servicio de la sociedad como tal, no hay ninguna sociedad en la que no haya formas de educación (Quintana, 1989).

\subsection{Carencia de predictibilidad social y comunitaria.}

Finalmente, encontramos un último elemento (en esta relación posiblemente incompleta) que afecta claramente al nivel de estrés social del ámbito educativo y que es un rasgo de nuestra sociedad humana, que además tiene una acentuación notable en estos momentos ( $\mathrm{y}$ que también se ha dado en otras épocas históricas). Nos referimos a una difícil previsión de futuro a nivel social y comunitario. Qué pasará? Sin saberlo ni aproximadamente, se genera angustia. No tenemos herramientas que nos permitan hacer predicciones a promedio, no conocemos ni podemos prever ni la duración ni la intensidad ni la reiterada frecuencia de las situaciones sociales estresantes. Y esto emerge como un factor de ansiedad especialmente en el ámbito educativo, hacia la búsqueda de una respuesta adecuada. Crespo-Labrador (2003:36ss).

\section{DISCUSIÓN}

Podemos decir que en el entorno docente hay aspectos sociales y sociológicos que se interrelacionan con gran complejidad y que en la realidad cotidiana de los docentes hacen que estos experimenten fuertes presiones y contradicciones, que pueden generar una experiencia estresante (Riart, Arís, y otros, 2013). La realidad es siempre compleja y cuando se hace referencia a fenómenos sociales se hace muy difícil hacer la 
tarea de diseccionar y analizar todas las causas. Hemos querido reflexionar sobre las mayores controversias en la actualidad.

-Con la aparición de Internet se rompe la idea de una educación basada en la "transmisión de paquetes de conocimientos acumulados" para convertirse en un aprendizaje de cómo ser capaz de acceder en la red, y obtener el máximo provecho Hay que aprender a seleccionar la información y a proporcionar instrumentos para procesarla con criterios constructivos.

-La sociedad-red plantea grandes retos en el mundo educativo. Estamos ante una verdadera revolución social y educativa. Este nuevo paradigma educativo provoca que el docente no sepa exactamente como "enseñar" a sus alumnos y evidentemente acentúa el estrés, el personal, el de su colectivo y el estrés social general.

-Así mismo, se constata una crisis de valores. Nuevas generaciones con referentes mentales y laborales diferentes y actitudes sociales diferentes. Esto constituye un elemento importante en las nuevas generaciones que, en este punto pueden ser especialmente erráticas en normas, actitud e incluso valores sociales y laborales.

-Por último, nos referimos a una difícil previsión de futuro a nivel social y comunitario. Vivir en la actual sociedad del conocimiento (Castells, 2000) implica desarrollar nuevas habilidades y una actitud de aprendizaje continuado a lo largo de la vida. En este contexto es relevante la misión de los maestros para interpretar y proyectar el perfil competencial del entorno a los itinerarios formativos, integrar nuevas maneras de construir conocimiento y adecuar la calidad de la enseñanza.

-Si la educación quiere promover cambios sociales lo que tiene que hacer es educar personas: participativas, independientes, críticas, flexibles y creativas. Pero la escuela, y los profesores no son más que uno de los agentes socializadores, su acción depende y a la vez refleja totalmente de los rasgos de la sociedad. Los maestros no pueden hacer el trabajo sólos, pero sobre todo, no lo pueden hacer si están angustiados o estresados. Las instituciones, la administración y los propios docentes tienen que ser conscientes de la importancia de la prevención de la salud profesional.

Es preciso un esfuerzo para incrementar las intervenciones, que ayuden los docentes a afrontar con eficacia las tensiones del día a día y del futuro inmediato.

La escuela es cosa de todos y esto implica padres, familias, niños, instituciones, y claro, también maestros. Por eso hay que insistir en la utilización instrumental y contextualizada del conocimiento para entender mejor los problemas específicos (Riart, Aris 2013).

\section{REFERENCIAS}

Alonso, B., Lobato, F.J., Pinar, M.M. (2001) Relaciones en el Equipo de Trabajo. BCN: EDEBÉ

Althusser (1968) Montesquieu: la política y la historia. Barcelona: Ariel. 
Arís, N. (2008) El sindrome de Burnout en los docentes de educación Infantil y educación primaria en la zona del Vallés Occidental": http:/ / www.tdx.cat/TDX-1027108-171248

Arís, N. (2009) Burnout syndrome in educators. Electronic Journal of Researh in Educational Psychology 7(2) (num.18) (pp. 829-848) http:/ /investigacion-psicopedagogica.org/revista/

Arís, N. (2010) El cansament emocional en els docents. Dins J. Riart, A. Martorell. (Coord.) L'estrès laboral dels docents. Malalties que pot provocar i propostes terapèutiques. Cap. 4, pàgs 33-41.

Arís, N. (2010b). La educación emocional y la comunicación escolar. Vivat Academia, 113, Universidad Complutense de Madrid.

Baudelot, C., Establet, R. (1976). La escuela capitalista en Francia. Madrid: siglo XXI.

Bauman, Z. (2007) Temps líquids. BCN: Viena.

Bauman, Z. (2007). Els reptes de l'educació en la modernitat líquida. Barcelona: Arcàdia

Bourdieu, P. ; Passeron, J. C.(1973). Los estudiantes y la cultura. Buenoa Aires: Labor.

Bourdieu, P. ; Passeron, J. C (1977). La reproducción. Barcelona: Laia.

Bowles, S.; Gintis, H. (1981). La instrucción escolar en la América capitalista. Madrid: siglo XXI.

Canto Ortíz, J.M. (1998) Psicología de los grupos. Estructura y procesos. Málaga: Aljibe.

Castells, M. (2000). La Era de la información. Madrid: Alianza Editorial.

Castells, M. (2002) La galàxia Internet: Reflexions sobre Internet, empresa i societat. Barcelona: Plaza \& Janés. C

Castells, M. (2009) La era de la información. Vol. II. El poder de la identidad. Madrid: Alianza. (versió anterior de la trilogia en Mèxico: Siglo XXI Editores).

Castells, M. ; Tubella, I. (2007). La transició a la societat en xarxa. Barcelona: Ariel.

Crespo, M.; Labrador, F.J. (2003) Estrés. Madrid: Síntesis.

Gago, L., Escalante, E. (2001) Relacions en l'entorn de treball. BCN: McGraw Hill.

Gallego, C. (2008). Alfabetització matemàtica i comunitats escolars. Temps d'Educació, 34, 29-66.

Gil, F., Alcover de la Hera, C.M., García, M., Roda, R., i Rodríguez, F. (1999) Prácticas de psicología de los grupos. Madrid: Pirámide.

Gracia, E., Herrero, J., i Musitu, G. (2002). Evaluación de recursos y estresores psicosociales en la comunidad. Madrid: Síntesis.

Morin, E. (1999). Los siete saberes necesarios para la educación del futuro. UNESCO. 
Morin, E. (1999). La cabeza bién amueblada. Barcelona: Seix Barral

Morin, E. (2001) Tenir el cap clar per organitzar els coneixements i aprendre a viure. BCN: La Campana.

Morin, E. (2008). Vers l'abîme? Paris: l'Herne.

Pañellas, M. i Alguacil, M.(2010). La relació família-IES: un estudi des de la perspectiva del pares. Temps d'Educació, 37, 2n semestre 2009 (169-195)

Quintana, J. M. (1989). Sociología de la Educación. Madrid: Dykinson.

Riart i A. Martorell (Coord.) Els estressors laborals docents i programes pal liatius. Barcelona: ISEP. Cap. 19, pàg.: 229-241.

Riart, J. (2010) Idees bàsiques sobre l'estrès. RE. Realisme Existencial. 63, 5-7. Monogràfic sobre estrès vital, (juliol).

Riart, J. (2010b) L'estrès i la relaxació de les emocions. RE. Realisme Existencial. Núm.63, Pàgs. 19-22. Monogràfic sobre estrès vital, (juliol).

Riart, J. (2010c) L'estrès i la relaxació de les emocions. Tècniques. Dins, J. Riart, A. Martorell (Coord.) L'estrès laboral dels docents. Malalties que pot provocar i propostes terapèutiques. BCN: ISEP., Cap. 11, pàg. 113-126.

Riart, J. (2010-2011) Valors i món educatiu. Revista del COPC. Núm: 227, desembre 2010- gener 2011, Monogràfic de la secció de Psicologia de l’Educació. Pàgs. 16-21.

Riart, J. (Coord.), Arís, N., Virgili C., Alguacil M. (2013) L'estrès, mites i realitats. Registres, tests i programes antiestrès per a tothom. Barcelona:UOC

Sol i Mauri, N. (2009) Tècniques col lectives com a programa pal liatiu de l'estrès laboral docent. Dins J. Riart, A. Martorell. Els estressors laborals docents i programes pal liatius. BCN: ISEP. Cap. 21.

Shaw, M. (1983) Dinámica de grupo. Psicología de la conducta de los Pequeños Grupos. BCN: Herder.

Trianes, M.V., Blanca, M.J., Fernández-Baena, F.J., Escobar, M., Maldonado, E.F. (2012) Evaluación y tratamiento del estrès cotidiano en la infancia. Papeles del psicólogo, Vol. 33, 1. Enero-abril, 30-35.

\section{Núria Arís Redó}

Profesora Agregada Facultad de Educación de la Universidad Internacional de Catalunya. Coordinadora del Máster Oficial de Investigación en CCSS. Investigadora del Grupo de Investigación de Estresores Laborales Docentes del Colegio de Psicólogos de Barcelona. Dicho grupo obtuvo el Premio de ensayo pedagógico de la Fundación San Jose de Calassanz, "Joan Profitós, 2012" con el trabajo “El estrés, mitos y realidades. Registros, tests y programas antiestrés para todo el mundo". 
Arís Redo 